\title{
A highly transmissible tuberculosis outbreak: the importance of bars
}

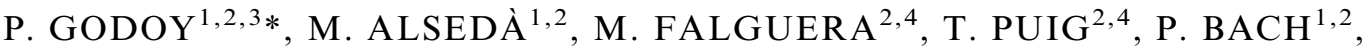 \\ M. MONRABÄ ${ }^{4}$ AND A. MANONELLES \\ ${ }_{2}^{1}$ Agència de Salut Pública de Catalunya, Barcelona, Spain \\ ${ }^{2}$ Institut de Recerca Biomèdica de Lleida, IRBLleida, Lleida, Spain \\ ${ }^{3}$ Ciber de Epidemiología y Salud Pública, CIBERESP, Madrid, Spain \\ ${ }^{4}$ Hospital Universitari Arnau de Vilanova, Lleida, Spain
}

Received 9 June 2017; Final revision 21 October 2017; Accepted 28 October 2017

\section{SUMMARY}

The transmission of tuberculosis (TB) in bars is difficult to study. The objective was to describe a large TB outbreak in a company's bar and other leisure settings. A descriptive study of a TB outbreak was carried out. Contacts were studied in the index case's workplace bar (five circles of contacts) and other recreational areas (social network of three bars in the index case's neighbourhood). Chest $\mathrm{X}$-rays were recommended to contacts with positive tuberculin skin tests (TST) $(\geqslant 5 \mathrm{~mm})$. The risk of latent tuberculosis infection (LTBI) was determined using an adjusted odds ratio. The dose-response relationship was determined using the chi-square test for linear trend. We studied 316 contacts at the index case's workplace and detected five new cases of TB. The prevalence of LTBI was $57 \cdot 9 \%$ (183/316) and was higher in the first circle, $96 \cdot 0 \%(24 / 25)$, and lower in the fifth, $46 \cdot 5 \%(20 / 43)(P<0 \cdot 0001)$. Among 58 contacts in the three neighbourhood bars, two TB cases were detected and the LTBI prevalence was $51 \cdot 7 \%$ (30/58). Two children of one secondary TB company patient became ill. Bars may be transmission locations for TB and, as they are popular venues for social events, should be considered as potential areas of exposure.

Key words: Bar, cavitary tuberculosis, contact investigation, epidemiology, latent tuberculosis infection.

\section{INTRODUCTION}

The estimated prevalence of infection among tuberculosis (TB) contacts in various studies is about $30 \%[1-4]$, much higher than that of the general population. The risk varies according to the characteristics of the index case and the duration and intensity of the exposure [3, 4]. The biggest determinant is the finding of smearpositive acid-fast bacilli (AFB) in respiratory

\footnotetext{
* Author for correspondence: P. Godoy, Epidemiological Surveillance Unit of Lleida, C/Alcalde Rovira Roure, 2, 25006 Lleida, Spain. (E-mail: pere.godoy@gencat.cat)
}

secretions from the index case, which are detected by microscopic examination [2, 5]. Cavitary lesions, generally associated with diagnostic delay, also have a higher volume and concentration of bacilli, which increase transmission [1, 3].

The concentric circles technique is recommended in investigations of contacts of TB patients, especially to prove the dose-response relationship between the level of exposure and the risk of latent tuberculosis infection (LTBI) [2]. It consists of beginning the investigation in the circle with the greatest exposure (persons living with patients or equivalent) and continuing to expand until a population group with no greater risk 
than the general population is reached $[2,3,6]$. To apply the technique correctly, close cooperation with the index case, in order to list all contacts, is required. However, when the prevalence of infection is very high and it is difficult to find an endpoint for testing, or when investigations must be extended to complex community environments, such as the customers of a bar, a social network approach may be recommendable $[7,8]$.

The aims of contact investigation are to detect associated cases, control sources of infection, reduce morbidity by detecting and treating LTBI and preventing future cases [9-11]. Fear of the stigma attached to the illness is a barrier to the complete, in-depth investigation of contacts [12]. Bars are meeting points and areas of social interaction in which contact investigations in TB cases are difficult $[13,14]$. The inability to identify and screen all at-risk contacts is one of the main reasons for new TB outbreaks [15].

On 19 March 2012, the Epidemiological Surveillance Unit of Lleida (Spain) was notified of a case of pulmonary TB. Lleida province is an area with 400000 inhabitants situated in the north-east of Spain, with a TB incidence rate of $19 \cdot 8$ new cases per $10^{5}$ people [16]. The patient was a 42-year-old non-immigrant male with symptoms of coughing and weight loss for several months. Examination showed bilateral lesions in the pulmonary parenchyma with multiple disseminated pseudonodular images with cavitary lesions in the chest radiography. The AFB and sputum culture were positive for Mycobacterium tuberculosis. The patient identified two family contacts, but categorically refused to identify other contacts in the business or social areas. After several unsuccessful attempts to obtain his cooperation over a 2-month period, the Epidemiological Unit finally made contact with the occupational health managers of the company where the patient worked.

The purpose of the investigation was to describe a high-transmission TB outbreak which occurred in bars and had to be investigated by applying both a social network approach and the concentric circles technique.

\section{METHODS}

We made a descriptive epidemiological study of the prevalence of LTBI and the detection of new TB patients at the index patient's workplace (company A), in the neighbourhood bars he frequented, and at the company where one of the secondary TB patients detected in the neighbourhood bars worked (company B).
A visual inspection of the index patient's workplace and the company A bar were conducted. Given the risk of transmission in the index patient's workplace, the occupational health service was contacted directly and investigation of employees at greatest risk (first circle) was recommended. A list of contacts was drawn up for study. A very high prevalence of infection was observed among these company workers. Therefore, five exposure circles were constructed in accordance with the level of exposure, based on the possibility of coinciding in the company bar (the only space shared by workers from different sections and shifts). The first circle consisted of employees working in the same section on the afternoon shift, where they were together with the patient on a daily basis and at the same time in the company bar for a period of about $30 \mathrm{~min}$. The second circle included employees in other sections who were also in contact with the patient every day at the same time in the bar. In the third circle, the study was extended to a group of employees in other sections who went to the bar every day, but after the index patient had left. The fourth circle consisted of workers on the morning and afternoon shifts who coincided with the index patient in the bar on a weekly, but not daily, basis. The fifth circle consisted of morning shift workers who coincided sporadically (less than weekly) with the index patient.

Further areas of exposure were studied via inspection and interviews with the index patient's social network and included three bars in the index patient's neighbourhood, the family members of all TB patients and company B.

All contacts were informed and offered a tuberculin skin test (TST) using the Mantoux method and PPD RT 23 with Tween 80 of $2 \mathrm{TU}$ per $0 \cdot 1 \mathrm{ml}$ [17]. A positive test was defined as a case in which the diameter of induration transverse to the long axis of the arm was equal to or $>5 \mathrm{~mm}$ after $72 \mathrm{~h}$ [17]. BCG vaccine scars were recorded. All contacts with a positive TST were offered chest radiography, a medical appointment and treatment for LTBI or TB if indicated.

The variables of the contacts investigated were: age, sex, place of exposure, country of origin (native or immigrant), circle of exposure in the index case's workplace, previous positive TB reaction, history of BCG vaccination, positive current tuberculin reaction, diameter of induration in millimetres and recommended LTBI treatment.

The following definitions were used: Household contacts were persons living in the same household 
as a TB patient. Contacts were included in the 'at risk' group for analysis if they had shared closed spaces with the index patient, attended the screening procedure and had not already been diagnosed with TB prior to the screening. People at risk in the neighbourhood bars were those who coincided weekly with the index TB patient and were identified by the bars' owners; this at-risk group was the denominator for estimating prevalence. Prevalent cases of TB among screened contacts were defined as previously-undiagnosed cases of TB among contacts diagnosed during the baseline contact investigation or within the first 6 months after diagnosis of the index patient. A case of TB was defined as bacteriologically confirmed TB (positive smear microscopy or cultures) or clinically diagnosed TB not fulfilling the criteria for bacteriological confirmation but diagnosed as active TB by a clinician who has decided to give the patient a full course of TB treatment. This definition includes cases diagnosed on the basis of X-ray abnormalities [17, 18]. Contacts diagnosed with LTBI or TB prior to the contact investigation were not considered prevalent cases, as they could not be attributed to the contact investigation.

The TST induration was described as the mean size and standard deviation (SD). The relationship between TST induration and the history of BCG vaccination was determined using an ANOVA test with a significance level of $P<0 \cdot 05$.

The main frequency measure was the prevalence of LTBI, and its association with sex, age group, immigration history, place of exposure and circle was determined using the odds ratio (OR) and 95\% confidence intervals $(\mathrm{CI})$. The dose-response relationship between the degree of exposure (circle) and the risk of LTBI was determined using the chi-square $\left(\chi^{2}\right)$ test for linear trend with a significance level of $P<$ $0 \cdot 05$. The adjusted OR (aOR) was estimated by multivariate analysis using conditional logistic regression. The analysis according to sex, age group and immigration history was adjusted using conditional logistic regression. Covariates were introduced into the model using a backward stepwise procedure, with a cut-off point of $P<0 \cdot 2$. The EpiInfo v7 statistical program was used.

As the study was undertaken as part of a public health outbreak response and was an official outbreak investigation, ethics approval was not required and data collation was conducted under regulations covering information collection.

\section{RESULTS}

In total, 404 of the 414 registered contacts were tested $(97 \cdot 6 \%)$. The average age was 38.1 years $( \pm 11 \cdot 7)$ and $29.0 \%$ were female. Contacts were distributed according to the following fields of exposure: 316 in the index case's workplace (company A), 58 in the three neighbourhood bars, 18 relatives of cases and 12 at the secondary case's workplace (company B).

The index case was detected on 19 March 2012. Over the next 7 months, nine further cases were identified (Fig. 1). There was a diagnostic delay of more than 6 months in identifying the index case (3 months due to the patient's reluctance to consult a doctor and 3 months due to the doctor not considering TB), who had cavitary lesions and a smear-positive sputum test. The patient refused to identify at-risk contacts. The second TB patient, who had pleural $\mathrm{TB}$, was a customer of the same neighbourhood bars that the index case frequented and identified three bars in the neighbourhood where the index case often went. Five cases appeared in the company where the index patient worked (prevalence $1 \cdot 6 \%, 5 /$ 316). Two children, aged 2 and 6 years, respectively, of the fifth patient from company A also became ill (prevalence between relatives of $11 \%, 2 / 18$ ). A second TB patient was identified among customers of the neighbourhood bars $(2 \cdot 3 \%, 2 / 58)$ (Table 1). No TB cases were detected in the secondary TB patient's workplace $(0 / 12)$.

The global prevalence of infection among all contacts identified was $55 \cdot 4 \%$ (224/404). The mean reaction to the TST was $18.2 \mathrm{~mm}( \pm 8)$, and was very similar in persons with BCG vaccination $(18 \cdot 2 \pm 8)$ and those without $(18 \pm 7 \cdot 7)(P<0.05)$. The prevalence in the different fields of exposure was $57.9 \%$ $(183 / 316)$ in the index case's workplace; $51 \cdot 7 \%(30 / 58)$ in the three neighbourhood bars; $38.9 \% \quad(7 / 18)$ among relatives of the cases; and $33 \cdot 3 \%(4 / 12)$ in the secondary case's workplace (Table 2).

Company A, where the index patient worked, is a well-known agricultural business that exports different kinds of sauces around Europe. The company bar is a very crowded place measuring approximately $80 \mathrm{~m}^{2}$. Seventy-five workers coincided there for 30 min at break-times for each shift. The space was poorly ventilated, especially in the winter months when the main exposure occurred. Prevalence rates of infection were very high, varying from $96 \%$ (24/ 25 ) in the first circle to $46 \cdot 5 \%(20 / 43)$ in the fifth circle. A dosage-response relationship was observed for 


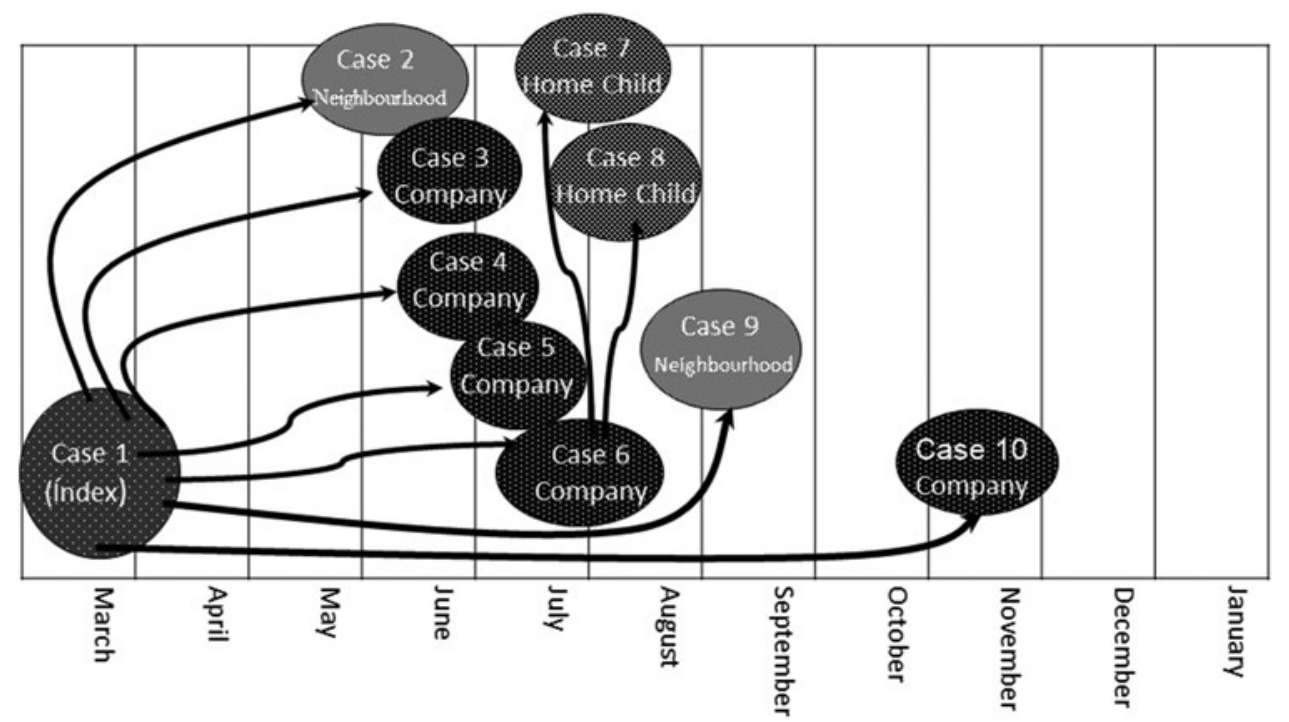

Fig. 1. Epidemic curve of a tuberculosis outbreak in Lleida, Spain.

LTBI according to the degree of exposure. The prevalence of LTBI was higher in immigrant workers $(74 \cdot 7 \%)$ than in native workers $(52 \cdot 7 \%)$, and this difference was similar in the five circles tested. Thus, the risk determined by logistic regression adjusted by sex, age and immigration history was much higher in the first $(\mathrm{aOR}=30 \cdot 5)$, second $(\mathrm{aOR}=3.6)$ and third $(\mathrm{aOR}=1.9)$ circles compared with the fifth circle (chi-square test for linear trend $=25 \cdot 1 ; P>0 \cdot 0001$ ) (Table 3).

\section{DISCUSSION}

An investigation of 404 persons at risk from a patient with cavitary TB was carried out using a social network approach and the concentric circles system. Nine cases of TB were detected, with a high prevalence of LTBI $(55.4 \%)$ and a dose-response effect between persons at risk in the index case's workplace bar. The investigation was time consuming due to a lack of cooperation from the index patient. Nevertheless, as it resulted in the detection and treatment of nine new cases of TB and 224 of LTBI, it was considered effective.

The investigation had some particular features. The index case showed the classic characteristics of cases which generate large outbreaks of TB: a delay in diagnosis, cavitary lesions and AFB smear-positive sputum [19]. There was also a relative delay in the study of contacts due to the index case's repeated refusal to identify them $[12,20,21]$. Strategies to reduce the stigma attached to $\mathrm{TB}$ and to improve interview systems with patients should be considered in order to prevent delays and make the investigation of contacts more comprehensive [21]. One of the secondary cases in the company caused two further cases in his children, aged 2 and 6 years, which also underlines how important it is to carry out investigations on a forward-looking and thorough basis so as to avoid transmission beyond the index case [19]. Two cases were detected in customers of the neighbourhood bars, showing that it is important to study these recreational areas and extend the studies to patients' social networks [8, 13, 14].

The global prevalence of infection $(55.4 \%)$ was higher than that documented in other contact investigations, which are generally about $30 \%[1,2,22]$. The prevalence was even higher in the first circle studied $(96 \%)$, with considerable exposure in the company bar, and this warranted the investigation being extended to other possible at-risk circles [3, 23].

The determining factor of the high prevalence was the estimated delay of over 6 months in diagnosing the index case. There was also a 2-month delay in starting the contact investigation, given the index case's refusal to collaborate. The delay resulted in prolonged exposure to his contacts, as shown by other investigations [19, 21]. As the TST was carried out more than 2 months after the last exposure to the index patients, it was not considered necessary to repeat the test in negative-TST contacts [3].

Despite the practical difficulties, the results obtained in the five circles constructed in the company are consistent with the differing levels of prevalence of 
Table 1. Characteristics of patients in a tuberculosis outbreak in Lleida (Spain)

\begin{tabular}{|c|c|c|c|c|c|c|c|c|}
\hline Case & Age & Sex & Symptoms started & Immigrant & Chest X-ray & Microbiology & Treatment started & Place of exposure \\
\hline 1 & 42 & M & 6-month delay & No & Cavitary & $\begin{array}{l}\text { Bk }+ \\
\text { Culture }+\end{array}$ & $19 / 3 / 12$ & Company A \\
\hline 2 & 50 & M & $12 / 5 / 12$ & No & $\begin{array}{l}\text { Abnormal } \\
\text { Non-cavitary }\end{array}$ & $\begin{array}{l}\text { BK- } \\
\text { Culture- }\end{array}$ & $14 / 6 / 12$ & Neighbourhood bar \\
\hline 3 & 32 & $\mathrm{~F}$ & $25 / 6 / 12$ & No & $\begin{array}{l}\text { Abnormal } \\
\text { Non-cavitary }\end{array}$ & $\begin{array}{l}\text { BK- } \\
\text { Culture- }\end{array}$ & $27 / 6 / 12$ & Company A \\
\hline 4 & 33 & M & $27 / 6 / 12$ & No & $\begin{array}{l}\text { Abnormal } \\
\text { Non-cavitary }\end{array}$ & $\begin{array}{l}\mathrm{BK}+ \\
\text { Culture + }\end{array}$ & $27 / 6 / 12$ & Company A \\
\hline 5 & 34 & M & No symptoms & No & $\begin{array}{l}\text { Abnormal } \\
\text { Non-cavitary }\end{array}$ & $\begin{array}{l}\text { BK- } \\
\text { Culture + }\end{array}$ & $10 / 7 / 12$ & Company A \\
\hline 6 & 40 & M & $3 / 6 / 12$ & Yes & Cavitary & $\begin{array}{l}\mathrm{BK}+ \\
\text { Culture }+\end{array}$ & $16 / 7 / 12$ & Company A \\
\hline 7 & 6 & $\mathrm{~F}$ & No symptoms & No & $\begin{array}{l}\text { Abnormal } \\
\text { Non-cavitary }\end{array}$ & $\begin{array}{l}\text { BK - } \\
\text { Culture- }\end{array}$ & $26 / 7 / 12$ & $\begin{array}{l}\text { Home } \\
\text { Child case } 6\end{array}$ \\
\hline 8 & 2 & $\mathrm{~F}$ & No symptoms & No & $\begin{array}{l}\text { Abnormal } \\
\text { Non-cavitary }\end{array}$ & $\begin{array}{l}\text { BK - } \\
\text { Culture - }\end{array}$ & $26 / 7 / 12$ & $\begin{array}{l}\text { Home } \\
\text { Child case } 6\end{array}$ \\
\hline 9 & 56 & M & $15 / 5 / 12$ & No & Cavitary & $\begin{array}{l}\mathrm{BK}+ \\
\text { Culture + }\end{array}$ & 24/8/12 & $\begin{array}{l}\text { Neighbourhood bar } \\
\text { Works for company B }\end{array}$ \\
\hline 10 & 33 & $\mathrm{~F}$ & $7 / 11 / 12$ & No & $\begin{array}{l}\text { Abnormal } \\
\text { Non-cavitary }\end{array}$ & $\begin{array}{l}\text { BK - } \\
\text { Culture - }\end{array}$ & $7 / 11 / 12$ & Company A \\
\hline
\end{tabular}

Table 2. Prevalence of tuberculosis (TB) infection in different sites of exposure to a case of $T B$ with cavitary lesions, Lleida (Spain) $(n=404)$

\begin{tabular}{ll}
\hline \hline Groups & $\begin{array}{l}\text { Prevalence of TB infection } \\
\%(\mathrm{n} / \mathrm{N})\end{array}$ \\
\hline Company A & $57 \cdot 9(183 / 316)$ \\
Bars in the neighbourhood & $51 \cdot 7(30 / 58)$ \\
All family members & $38 \cdot 9(7 / 18)$ \\
Company B & $33 \cdot 3(4 / 12)$ \\
\hline \hline
\end{tabular}

the infection $(96 \%, 73 \cdot 6 \%, 58 \cdot 9 \%, 48 \cdot 2 \%$ and $46 \cdot 5 \%)$. The first circle of persons at risk included the work contacts of patients who, due to the intensity of exposure (hours and volume of shared air) can be considered similar to cohabiting contacts. A visual inspection of the workplace revealed a bar often visited by the index case, which was the only space shared with other employees. We were thus able to assess the risk of this space according to the number of visits to the bar and the time shared with the index case. Guidelines on contact investigations expressly recommend the inspection and direct observation of places of exposure, particularly in outbreaks [3], as they may provide very valuable information which can be used to prioritise the contacts to be studied $[2,23]$. In this way, we were able to estimate an important dose-response relationship between the time of exposure in the bar of company A and the risk of LTBI as a criterion of transmission. Nevertheless, other techniques such as the social network approach should be recommended, especially in venues with a high prevalence of LTBI and where it may be difficult to identify everyone who may have been exposed [8].

There is considerable evidence about the presence of customers affected by TB in bars [13, 14, 24-27]. The investigation confirmed that the index patient had spent an average of $6 \mathrm{~h}$ a week in three bars in his neighbourhood. Through interviews with the bars' owners, 58 habitual customers were located and their possible exposure evaluated. The global prevalence of LTBI observed in customers of the three bars $(51.7 \%)$ was high and similar to that observed in other investigations [13, 14, 24, 25]. Bars are very popular establishments in Spain and should be evaluated and, if possible, studied in contact investigations by applying a social network approach where necessary.

We made great efforts to communicate with persons at risk, and the companies and bars involved. Communication must be timely, clear and transparent [12], as it is a crucial way of obtaining an exhaustive list of contacts and, subsequently, adequately investigating and monitoring all persons affected [12, 21, 28]. The percentage of coverage of the investigation $(97.6 \%)$ must be considered positive [2, 29]. Treatment for 
Table 3. Variables associated with a tuberculosis (TB) outbreak in the bar of company A, Lleida (Spain) ( $n=316)$

\begin{tabular}{|c|c|c|c|c|c|}
\hline Groups & $\begin{array}{l}\text { Prevalence of TB } \\
\%(n / N)\end{array}$ & OR & $95 \% \mathrm{CI}$ & $\mathrm{aOR}$ & $95 \% \mathrm{CI}$ \\
\hline \multicolumn{6}{|l|}{ Sex } \\
\hline Male & $60 \cdot 6(137 / 226)$ & $1 \cdot 5$ & $0 \cdot 9-2 \cdot 5$ & $1 \cdot 4$ & $0 \cdot 8-2 \cdot 4$ \\
\hline Female & $50 \cdot 6(45 / 89)$ & $1 \cdot 0$ & & $1 \cdot 0$ & \\
\hline \multicolumn{6}{|l|}{ Age (years) } \\
\hline $15-24$ & $42 \cdot 1(8 / 19)$ & $1 \cdot 0$ & - & $1 \cdot 0$ & - \\
\hline $25-39$ & $55 \cdot 7(93 / 167)$ & $1 \cdot 7$ & $0 \cdot 7-4 \cdot 7$ & $1 \cdot 6$ & $0 \cdot 6-4 \cdot 7$ \\
\hline $40-54$ & $62 \cdot 2(74 / 119)$ & $2 \cdot 3$ & $0 \cdot 9-6 \cdot 0$ & $2 \cdot 5$ & $0 \cdot 8-7 \cdot 3$ \\
\hline$>54$ & $75 \cdot 0(9 / 12)$ & $4 \cdot 1$ & $0 \cdot 8-20 \cdot 3$ & $5 \cdot 7$ & $1 \cdot 1-30 \cdot 7$ \\
\hline \multicolumn{6}{|c|}{ Country of origin } \\
\hline Native & $52 \cdot 7(127 / 241)$ & $1 \cdot 0$ & & $1 \cdot 0$ & \\
\hline Immigrant & $74 \cdot 7(56 / 75)$ & $2 \cdot 6$ & $1 \cdot 5-4 \cdot 7$ & $2 \cdot 8$ & $1 \cdot 5-5 \cdot 3$ \\
\hline \multicolumn{6}{|c|}{ Contact circle* } \\
\hline First & $96 \cdot 0(24 / 25)$ & $27 \cdot 6$ & $3 \cdot 4-222 \cdot 7$ & $30 \cdot 5$ & $3 \cdot 7-249 \cdot 6$ \\
\hline Second & $73 \cdot 6(39 / 53)$ & $3 \cdot 2$ & $1 \cdot 4-7 \cdot 5$ & $3 \cdot 6$ & $1 \cdot 5-8 \cdot 6$ \\
\hline Third & $58 \cdot 9(33 / 56)$ & $1 \cdot 7$ & $0 \cdot 7-3 \cdot 7$ & 1.9 & $0 \cdot 8-4 \cdot 4$ \\
\hline Fourth & $48 \cdot 2(67 / 139)$ & $1 \cdot 1$ & $0 \cdot 5-2 \cdot 1$ & $1 \cdot 1$ & $0 \cdot 5-2 \cdot 1$ \\
\hline Fifth & $46 \cdot 5(20 / 43)$ & $1 \cdot 0$ & & $1 \cdot 0$ & \\
\hline Total & $57 \cdot 9(183 / 316)$ & & & & \\
\hline
\end{tabular}

OR, odds ratio; CI, confidence intervals; aOR, adjusted odds ratio.

$* \chi^{2}$ of linear trend $=25 \cdot 1 ; P>0.0001$ (contact circle).

LTBI was recommended to $42 \cdot 6 \%$ of the people studied $(171 / 401)$ and was completed by $82 \cdot 5 \%(52 / 63)$ of those who were provided with this information, which is similar to the results reported by other studies [9].

The investigation had some limitations. As the laboratory did not retain the Mycobacterium tuberculosis culture, we could not genotype the strains and were therefore unable to identify any unrelated cases, although the probability of this was very low. The list of contacts and the construction of the persons-at-risk circles were prepared based on a visual inspection of the areas of exposure and the interviews held with the company owners, secondary cases and bar owners. Some alarm was generated in these places, and had to be addressed by specific efforts at communication. Due to these circumstances, some persons may have been classified as having a higher risk when in fact they had a lesser degree of exposure, which could have helped to reduce the LTBI prevalence observed [13]. However, the prevalence observed in the fifth contact circle was still very high. Some positive TST may have been due to interference by BCG vaccines, although the reactions to the TST were very similar in persons vaccinated or not with BCG $(18 \cdot 2 \pm 8$ vs. $18 \pm 7 \cdot 7)$. The TST reaction due to the booster effect of BCG is around $5-10 \mathrm{~mm}$, and thus we believe most positive TST were due to true infection. However, we could not rule out some residual confounding effect of BCG, particularly among immigrants [3]. There is no single criterion that identifies contacts vaccinated with BCG as infected and, in practice, different cut-off points have been used $[3,30]$, showing how difficult it is to determine the effect of BCG on the TST [30]. To detect this effect, interferon-gamma release assays should be used in these patients in order to detect false positives and avoid unnecessary treatment [31].

The investigation has some implications. TB with extensive cavitary lesions due to diagnostic delay implies high transmission levels among contacts. To ensure a comprehensive investigation, the risk population's circles should be expanded until low levels of prevalence are found $[1,2]$. This requires direct cooperation from the index patient, the institutions involved, and a visual inspection of the sites of exposure $[3,21]$. TB infection is still stigmatised $[12,21]$ and, as a result, the index case may be unwilling to cooperate in the contact investigation [21]. Strategies should be identified to lessen the stigma associated with TB and improve interview methods $[12,21]$. Techniques such as the social network approach should be recommended especially in locations where it may be difficult to identify all persons exposed [8]. 


\section{CONCLUSIONS}

Bars, which may be transmission sites, are popular in Spain, and should be considered as potential areas of exposure in the case of cavitary TB among employees and customers. Transmission in bars should be investigated by applying both the social network approach and concentric circles techniques. The possible sense of alarm generated in these cases should be addressed by providing the affected groups with swift, complete and transparent information.

\section{ACKNOWLEDGEMENTS}

This study was supported by the Catalan Agency for the Management of Grants for University Research (AGAUR Grant number 2014/SGR1403).

\section{DECLARATION OF INTEREST}

No author has any commercial or other association that might pose a conflict of interest.

\section{REFERENCES}

1. Horsburgh CR, Rubin EJ. Latent tuberculosis infection in the United States. New England Journal of Medicine 2011; 364: 1441-1448.

2. Fox GJ, et al. Contact investigation for tuberculosis: a systematic review and meta-analysis. European Respiratory Journal 2013; 41: 140-156.

3. National Tuberculosis Controllers Association; Centers for Disease Control and Prevention (CDC). Guidelines for the investigation of contacts of persons with infectious tuberculosis. Recommendations from the National Tuberculosis Controllers Association and CDC. MMWR. Recommendations and Reports: Morbidity and Mortality Weekly Report. Recommendations and Reports United States, 2005; 54: 1-47.

4. Alsedà M, Godoy $\mathbf{P}$. Factores asociados a la infección tuberculosa latente en los contactos de pacientes afectados. Gaceta Sanit 2004; 18: 101-107.

5. Godoy $\mathbf{P}$, et al. Characteristics of tuberculosis patients with positive sputum smear in Catalonia, Spain. European Journal of Public Health 2004; 14: 71-75.

6. Veen J. Microepidemics of tuberculosis: the stonein-the-pond principle. Tubercle and Lung Disease: International Union of Tuberculosis and Lung Disease 1992; 73: 73-76.

7. McElroy PD, et al. A network-informed approach to investigating a tuberculosis outbreak: implications for enhancing contact investigations. International Journal of Tuberculosis and Lung Disease 2003; 7: 486-493.

8. Fitzpatrick LK, et al. A preventable outbreak of tuberculosis investigated through an intricate social network. Clinical Infectious Diseases 2001; 33: 1801-1806.
9. Horsburgh CR, et al. Latent TB infection treatment acceptance and completion in the United States and Canada. Chest 2010; 137: 401-409.

10. Erkens CGM, et al. Risk of developing tuberculosis disease among persons diagnosed with latent tuberculosis infection in the Netherlands. European Respiratory Journal 2016; 48: 1420-1428.

11. Wejse C. Tuberculosis elimination in the post Millennium Development Goals era. International Journal of Infectious Diseases 2015; 32: 152-155.

12. Chang S, Cataldo JK. A systematic review of global cultural variations in knowledge, attitudes and health responses. International Journal of Tuberculosis and Lung Disease 2014; 18: 168-173.

13. Godoy $\mathbf{P}$, et al. Estudio de contactos según círculos concéntricos en un caso de tuberculosis laríngea. Gaceta Sanitaria 2013; 27: 279-281.

14. Kline SE, Hedemark LL, Davies SF. Outbreak of tuberculosis among regular patrons of a neighborhood bar. The New England Journal of Medicine 1995; 333: 222-227.

15. Mitruka K, et al. Tuberculosis outbreak investigations in the United states, 2002-2008. Emerging Infectious Diseases 2011; 17: 425-431.

16. Rodés-Monegal A, et al. La tuberculosi a Catalunya l'any 2014. Butlletí Epidemiologic de Catalunya 2016; 37: 186-195.

17. Centro Nacional de Epidemiología. Instituto de Salud Carlos III. Protocolos de la red nacional de vigilancia epidemiológica. 2015; 84-99.

18. World Health Organisation. Definitions and Reporting Framework for Tuberculosis -2013 Revision. Geneva: World Health Organisation, 2014, pp. 1-40.

19. Haddad MB, et al. Characteristics of tuberculosis cases that started outbreaks in the United States, 2002-2011. Emerging Infectious Diseases 2015; 21: 508-510.

20. Ospina JE, et al. Community health workers improve contact tracing among immigrants with tuberculosis in Barcelona. BMC Public Health 2012; 12: 158.

21. Faccini M, et al. Tuberculosis-related stigma leading to an incomplete contact investigation in a low-incidence country. Epidemiology and Infection 2015; 143: 28412848.

22. Erkens CGM, et al. Monitoring latent tuberculosis infection diagnosis and management in the Netherlands. European Respiratory Journal, 2016; 47: 1492-1501.

23. Erkens CGM, et al. Tuberculosis contact investigation in low prevalence countries: a european consensus. European Respiratory Journal 2010; 36: 925-949.

24. Bolam B, et al. Tuberculosis outbreak linked to a pub in North Somerset, England. Euro Surveillance, 2007; 12: e070322·3.

25. Nakamura Y, et al. A small outbreak of pulmonary tuberculosis in non-close contact patrons of a bar. Internal Medicine 2004; 43: 263-267.

26. Ijaz K, et al. Mycobacterium tuberculosis transmission between cluster members with similar fingerprint patterns. Emerging Infectious Diseases 2002; 8: 1257-1259.

27. Pettit S, et al. Outbreak of tuberculosis at a Newcastle public house: the role and effectiveness of contact 
screening. Communicable Disease and Public Health England, 2002; 5: 48-53.

28. Sandgren A, et al. Initiation and completion rates for latent tuberculosis infection treatment: a systematic review. BMC Infectious Diseases, 2016; 16: 204.

29. Mulder C, et al. Coverage and yield of tuberculosis contact investigations in the Netherlands. International Journal of Tuberculosis and Lung Disease 2011; 15: 1630-1636.
30. Farhat M, et al. False-positive tuberculin skin tests: what is the absolute effect of BCG and non-tuberculous mycobacteria? International Journal of Tuberculosis and Lung Disease 2006; 10: 1192-1204.

31. Santin M, et al. Executive summary of the guidelines for the use of interferon-gamma release assays in the diagnosis of tuberculosis infection. Enfermedades Infecciosas y Microbiologia Clinica, 2016; 34: 304-308. 